\title{
Simple Polygons of Maximum Perimeter Contained in a Unit Disk
}

\author{
Charles Audet • Pierre Hansen · Frédéric Messine
}

Received: 18 September 2007 / Revised: 2 May 2008 / Accepted: 26 May 2008 /

Published online: 20 June 2008

(C) Springer Science+Business Media, LLC 2008

\begin{abstract}
A polygon is said to be simple if the only points of the plane belonging to two of its edges are its vertices. We answer the question of finding, for a given integer $n$, a simple $n$-sided polygon contained in a disk of radius 1 that has the longest perimeter. When $n$ is even, the optimal solution is arbitrarily close to a line segment of length $2 n$. When $n$ is odd, the optimal solution is arbitrarily close to an isosceles triangle.
\end{abstract}

Keywords Simple polygon $\cdot$ Perimeter

\section{Introduction}

In this paper, we give a complete answer to the question posed by Brass, Moser and Pach [1, p. 437]: for a given integer $n$, find a simple $n$-sided planar polygon contained in a disk of radius 1 that has the longest perimeter. A polygon is said to be simple, or nonintersecting, if the only points of the plane belonging to two of its edges are its vertices. These authors mention that the optimal solution for the case where $n$ is even is arbitrarily close to the trivial upper bound $2 n$. It suffices to consider a simple

Work of the first author was supported by NSERC grant 239436-05, AFOSR FA9550-07-1-0302, and ExxonMobil. Work of the second author was supported by NSERC grant 105574-02.

C. Audet $(\varangle) \cdot$ P. Hansen

GERAD and Département de Mathématiques et de Génie Industriel, École Polytechnique de Montréal, C.P. 6079, Succ. Centre-ville, Montréal, Québec, H3C 3A7 Canada

e-mail: Charles.Audet@gerad.ca

P. Hansen

e-mail: Pierre.Hansen@gerad.ca

F. Messine

ENSEEIHT-IRIT, UMR-CNRS 5505, 2 rue Camichel, BP 7122, 31071 Toulouse Cedex 7, France

e-mail: Frederic.Messine@n7.fr 
polygon with all vertices with odd indices being arbitrarily close to $(-1,0)$ and all vertices with even indices being arbitrarily close to $(1,0)$.

We show that for $n=3$, the optimal solution is the equilateral triangle and that for odd values of $n \geq 5$, the optimal solution is arbitrarily close to an isosceles triangle.

\section{A Simple Polygon with Longest Perimeter}

Let $n \geq 3$ be an odd integer, and $v_{1}, v_{2}, \ldots, v_{n}$ denote the $n$ consecutive vertices of a simple polygon contained in a disk of radius 1 . Define $N=\{1,2, \ldots, n\}$, and for any $i \in N$, let $c_{i}=\left\|v_{i+1}-v_{i}\right\|$ be the Euclidean distance between the two consecutive vertices $v_{i}=\left(x_{i}, y_{i}\right)$ and $v_{i+1}=\left(x_{i+1}, y_{i+1}\right)$ (throughout this note, sums of indices are taken modulo $n$ ). The perimeter of the polygon may then be written as $p=\sum_{i=1}^{n} c_{i}$.

Define $p_{n}^{*}$ to be the supremum of the perimeter in value over all simple polygons contained in a disk of radius 1 . We use the term supremum instead of maximum since we will see that there are no optimal simple polygons when $n>3$. Indeed, we already know that the optimal solution for an even number of vertices is arbitrarily close to a line segment, a figure with only 2 vertices. For $n=3$, an equilateral triangle yields a lower bound of $\underline{p}_{3}=3 \sqrt{3}$ on $p_{3}^{*}$. For an odd number of vertices $n \geq 5$, one can easily show that $\underline{p}_{n}=2(n-2)+2 \sqrt{2}=2 n-2(2-\sqrt{2})>\sqrt{3} n$ is a lower bound on $p_{n}^{*}$. To do so, it suffices to construct a polygon with all of its vertices with even index arbitrarily close to $(1,0)$, the vertex $v_{n}$ at $(0,1)$, and the remaining ones arbitrarily close to $(-1,0)$. These lower bounds on $p_{n}^{*}$ are not tight since a small displacement of all vertices at $( \pm 1,0)$ on the circle (and away from $(0,1))$ produces a new isosceles triangle. Then the derivative of the length of the edges between $(-1,0)$ and $(1,0)$ is zero, and the derivative of the length of the edges between $(0,1)$ and $( \pm 1,0)$ is strictly positive. Therefore, a small displacement will increase the perimeter, and we will show that the optimal figure is obtained by such a displacement.

By relabeling the indices if necessary, we suppose that $n$ and 1 are the indices of the consecutive vertices that are the farthest apart, i.e., $c_{n} \geq c_{i}$ for all $i \in N$. Furthermore, since we are interested in maximizing the perimeter, we will consider that $c_{n} \geq \frac{p_{n}}{n}$. In particular, this implies that $c_{3} \geq \sqrt{3}$ and that $c_{n} \geq 2-\frac{2}{n}(2-\sqrt{2})>\sqrt{3}$ for odd $n \geq 5$. Furthermore, we will assume that the disk containing the polygon is centered at $(0,0)$ and that the vertices $v_{1}$ and $v_{n}$ are vertically aligned: $x_{n}=x_{1}$ and $y_{n}=y_{1}+c_{n}$.

Define

$$
U=\left\{(a, b): a^{2}+b^{2} \leq 1, b \geq c_{n}-1\right\}
$$

and

$$
L=\left\{(a, b): a^{2}+b^{2} \leq 1, b \leq 1-c_{n}\right\} .
$$

The next result shows that any two consecutive vertices of an optimal polygon cannot both lie in the same zone $L$ or $U$.

Lemma 1 If $n \geq 5$ and if two consecutive vertices $v_{\ell}$ and $v_{\ell+1}$ belong to same set $U$ or $L$, then $p<\underline{p}_{n}$. 
Fig. 1 Separation of the disk into zones

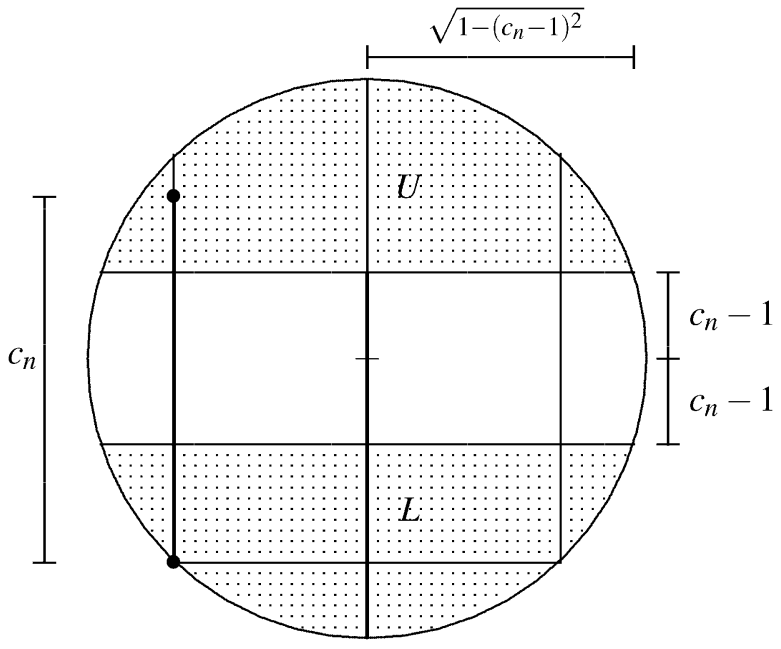

Proof If $v_{\ell}$ and $v_{\ell+1}$ are in the same set $L$ or $U$ (represented by the shaded regions of Fig. 1), then

$$
c_{\ell}=\left\|v_{\ell+1}-v_{\ell}\right\| \leq 2 \sqrt{1-\left(c_{n}-1\right)^{2}}=2 \sqrt{2 c_{n}-c_{n}^{2}}
$$

It follows that

$$
p=\sum_{i=1}^{n} c_{i} \leq(n-1) c_{n}+2 \sqrt{2 c_{n}-c_{n}^{2}} .
$$

The maximum of the right part of the previous inequality is attained either when $c_{n}$ is at one of its bound 2 or $\sqrt{3}$ or when the first derivative with respect to $c_{n}$ equals zero, i.e., when $(n-1)+2\left(1-c_{n}\right) / \sqrt{2 c_{n}-2 c_{n}^{2}}=0$. One can easily verify that the maximum occurs when $c_{n}=\frac{n-1}{\sqrt{n^{2}-2 n+5}}+1$, and therefore

$$
p \leq \sqrt{n^{2}-2 n+5}+n-1
$$

Furthermore, one can show that if $n \geq 5$, then this upper bound on the perimeter is strictly inferior to the lower bound $\underline{p}_{n}=2 n-4+2 \sqrt{2}$ since $n^{2}-2 n+5<$ $(n-3+2 \sqrt{2})^{2}$.

The next lemma shows that there are three consecutive vertices that satisfy an ordering property.

Lemma 2 There exist three consecutive vertices $v_{\ell-1}$, $v_{\ell}$, and $v_{\ell+1}$ for some $\ell \in$ $\{2,3, \ldots, n-1\}$ such that either $y_{\ell-1} \leq y_{\ell} \leq y_{\ell+1}$ or $y_{\ell-1} \geq y_{\ell} \geq y_{\ell+1}$.

Proof The previous lemma ensures that $y_{2}>1-c_{n} \geq y_{1}$ and $y_{n-1}<c_{n}-1 \leq y_{n}$. 
Fig. 2 Maximization of $c_{\ell-1}+c_{\ell}$ without intersecting $\overline{v_{1} v_{n}}$

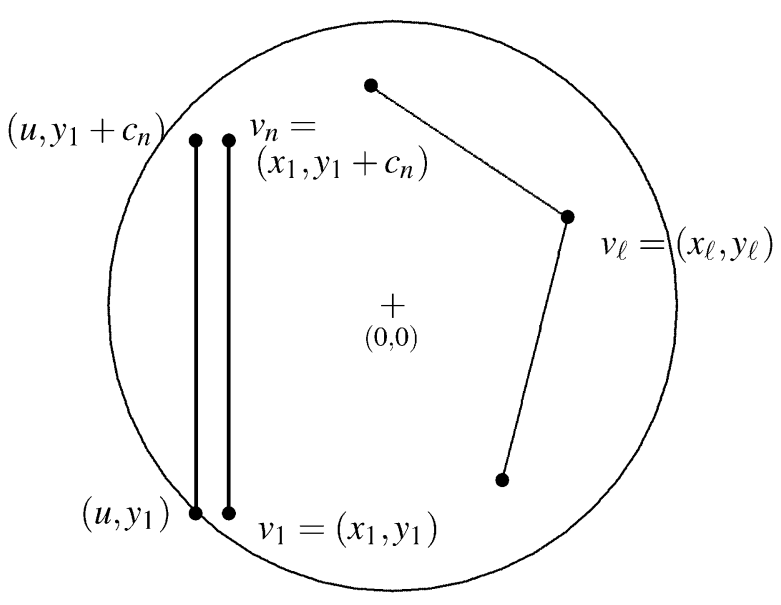

Suppose now that the result is false. Then, $y_{2}>y_{1}$ implies that $y_{3}<y_{2}$. Similarly $y_{3}<y_{2}$ implies that $y_{4}>y_{3}$. More generally, if $i$ is even, then $y_{i+1}<y_{i}$ and $y_{i}>y_{i-1}$. It follows that, since $n$ is odd, $y_{n-1}>y_{n}$, which is a contradiction.

Without loss of generality and by taking vertical and horizontal symmetrical images if necessary, we can assume that the vertex $v_{\ell}$ of Lemma 2 is such that $x_{\ell} \geq x_{1}$ and $\left|y_{1}\right| \geq\left|y_{n}\right|>0$ (i.e., that $-y_{1} \geq y_{1}+c_{n}>0$ ). Define $u \in\left[-\sqrt{1-c_{n}^{2} / 4}, 0\right]$ to be the smallest scalar such that $u^{2}+y_{1}^{2} \leq 1$. Then the segment joining $\left(u, y_{1}\right)$ and $\left(u, y_{1}+c_{n}\right)$ does not intersect the segments $\overline{v_{\ell-1} v_{\ell}}$ and $\overline{v_{\ell} v_{\ell+1}}$. These quantities are illustrated on Fig. 2.

The objective of the next results is to derive an upper bound on the sum $c_{\ell-1}+$ $c_{\ell}=\left\|v_{\ell}-v_{\ell-1}\right\|+\left\|v_{\ell}-v_{\ell+1}\right\|$. The two previous lemmas ensure that the vertex $v_{\ell}$ belongs to the set $\Omega=\left\{(a, b): u \leq a \leq 1-b^{2},|b| \leq 1-c_{n}\right\}$. Define the function $f: \mathbb{R} \rightarrow \mathbb{R}$ as

$$
f(x)=\operatorname{dist}\left(\left(x, y_{\ell}\right), v_{\ell-1}\right)+\operatorname{dist}\left(\left(x, y_{\ell}\right), v_{\ell+1}\right)
$$

for $\left(x, y_{\ell}\right) \in \Omega$. It is a convex function, and its maximum occurs when $\left(x, y_{\ell}\right)$ belongs to the boundary of $\Omega$, i.e., when $x=u$ or when $x=\sqrt{1-y_{\ell}^{2}}$. The next pair of lemmas considers these cases.

Lemma 3 If $n \geq 5$ and $v_{\ell}=\left(u, y_{\ell}\right)$, then $p<\underline{p}_{n}$.

Proof If $v_{\ell}=\left(u, y_{\ell}\right)$, then $c_{\ell-1}+c_{\ell} \leq-u+\sqrt{1-y_{\ell}^{2}}+\sqrt{u^{2}+y_{\ell}^{2}}+1$, as illustrated in Fig. 3.

It follows that

$$
\begin{aligned}
& \max _{y}-u+\sqrt{1-y^{2}}+\sqrt{u^{2}+y^{2}}+1 \\
& \text { s.t. } \quad u^{2}+y^{2} \leq 1
\end{aligned}
$$


Fig. 3 The vertex $v_{\ell}$ satisfies $x_{\ell}=u \leq 0$

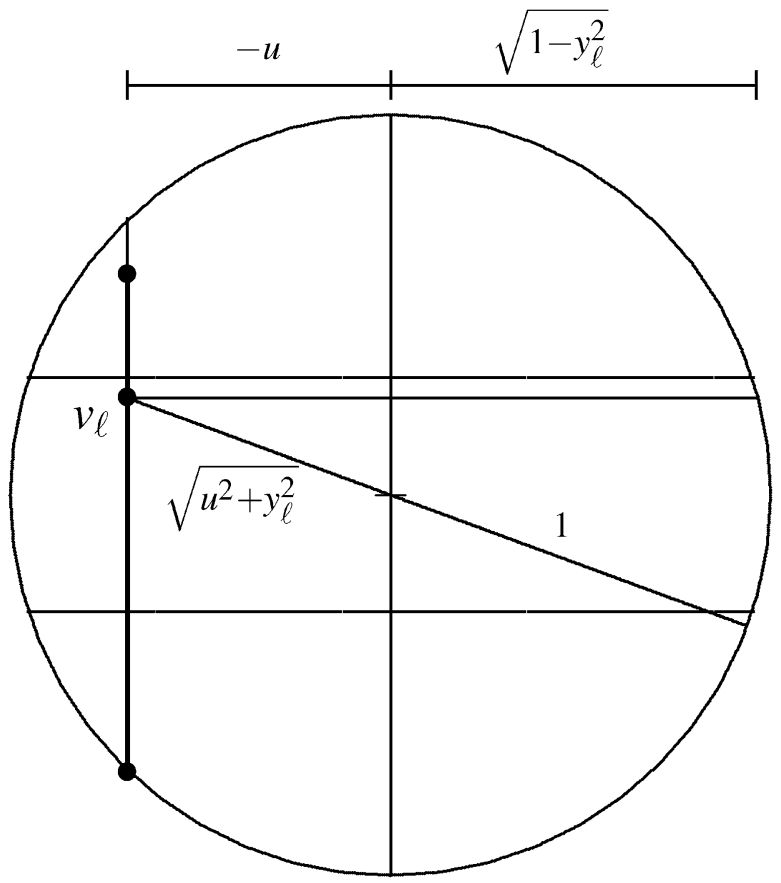

is a valid upper bound on $c_{\ell-1}+c_{\ell}$. The maximum is attained when $y^{2}=\frac{1}{2}\left(1-u^{2}\right)$, and the optimal value is $z(u)=-u+\sqrt{2+2 u^{2}}+1$. Using the facts that $-u \leq$ $\sqrt{1-c_{n}^{2} / 4}$ and $c_{n}>\sqrt{3}$ when $n \geq 5$, one can show that

$$
z(u)+c_{n}<\sqrt{1-3 / 4}+\sqrt{2+2(1-3 / 4)}+1+\sqrt{3}=3 / 2+\sqrt{5 / 2}+\sqrt{3} .
$$

Therefore

$$
\begin{aligned}
p & \leq(n-2) c_{n}+z(u)=(n-3) c_{n}+z(u)+c_{n} \\
& <2(n-3)+3 / 2+\sqrt{5 / 2}+\sqrt{3} \\
& =2 n-9 / 2+\sqrt{5 / 2}+\sqrt{3}<2 n-4+2 \sqrt{2}=\underline{p}_{n} .
\end{aligned}
$$

Lemma 4 If $n \geq 5$ and $v_{\ell}=\left(\sqrt{1-y_{\ell}^{2}}, y_{\ell}\right)$ or if $n=3$, then

$$
c_{\ell-1}+c_{\ell} \leq 2 \sqrt{2+2 \sqrt{1-\frac{c_{n}^{2}}{4}}}
$$

Proof Consider the case where $v_{\ell}=\left(\sqrt{1-y_{\ell}^{2}}, y_{\ell}\right)$.

Define $v_{1}^{\prime}=\left(u, y_{1}\right)$, and let $v_{n}^{\prime}=\left(x_{n}^{\prime}, y_{n}^{\prime}\right)$ be the point on the boundary of the disk such that $\left\|v_{n}^{\prime}-v_{1}\right\|=c_{n}$ and $x_{n}^{\prime} \leq u$. For any $0 \leq \theta \leq 2 \pi$, define $p(\theta)$ to be the point on the boundary of the circle that makes an angle of $\theta$ with $v_{\ell}$, as illustrated in the left part of Fig. 4. Then $p(0)=v_{\ell}$. Define $0 \leq \theta_{n}^{\prime} \leq \pi \leq \theta_{1}^{\prime} \leq 2 \pi$ to be the angles that satisfy $v_{1}^{\prime}=p\left(\theta_{1}^{\prime}\right)$ and $v_{n}^{\prime}=p\left(\theta_{n}^{\prime}\right)$. 

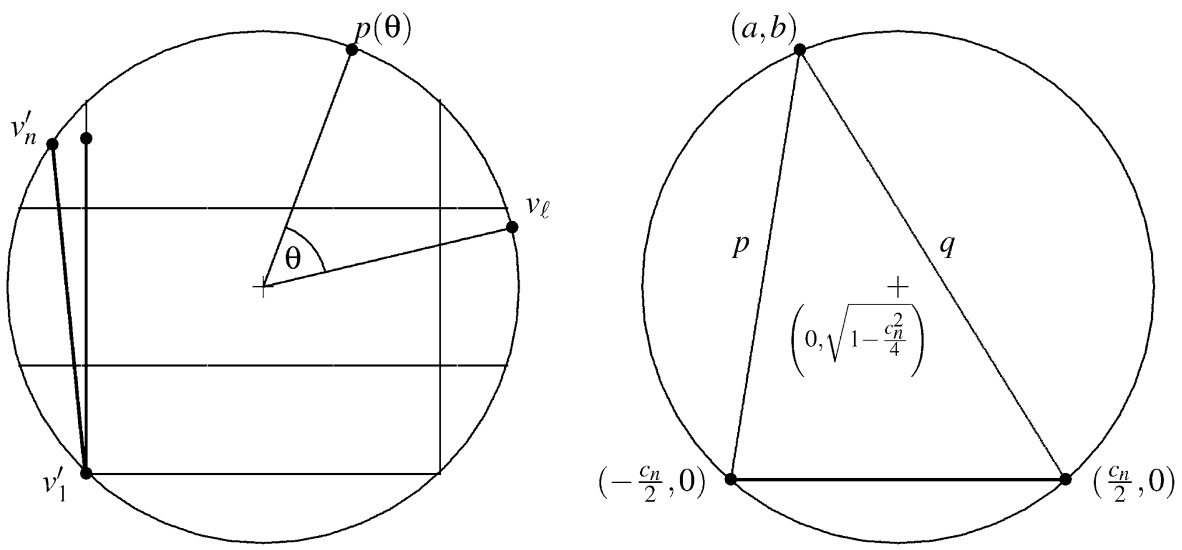

Fig. 4 The point on the disk at maximum distance from $\left(-\frac{c_{n}}{2}, 0\right)$ and $\left(\frac{c_{n}}{2}, 0\right)$

The distance function, $\left\|p(\theta)-v_{\ell}\right\|$ is increasing for $\theta \in[0, \pi]$ and decreasing for $\theta \in[\pi, 2 \pi]$. However, if $\theta_{n}^{\prime}<\theta<\theta_{1}^{\prime}$, then the segment joining $p(\theta)$ and $v_{\ell}$ intersects the segment $\overline{v_{1}^{\prime} v_{n}^{\prime}}$. It follows that the sum $c_{\ell-1}+c_{\ell}$ is bounded above by $\left\|v_{\ell}-v_{n}^{\prime}\right\|+$ $\left\|v_{\ell}-v_{1}^{\prime}\right\|$.

To simplify the presentation, let us move the disk so that the segment $\overline{v_{1}^{\prime} v_{n}^{\prime}}$ is horizontal with end points $\left( \pm c_{n} / 2,0\right)$, and the coordinates of $v_{\ell}$ become $(a, b)$ with $b \geq 0$, as illustrated in the right part of Fig. 4 . Observe that the case where $n=3$ is represented by the right side of that figure, as all three vertices of the optimal triangle necessarily lie on the boundary of the disk.

Equation (1) provides an upper bound on the sum:

$$
\begin{gathered}
\| \begin{array}{c}
(a, b)+\left(\frac{c_{n}}{2}, 0\right) \\
\leq \max _{a, b, p, q} p+q
\end{array} \text { s.t. } \quad p^{2}=\left(a+\frac{c_{n}}{2}\right)^{2}+b^{2}, \\
q^{2}=\left(a-\frac{c_{n}}{2}\right)^{2}+b^{2}, \\
a^{2}+\left(b-\sqrt{1-\frac{c_{n}^{2}}{4}}\right)^{2}=1 .
\end{gathered}
$$

The Lagrange multipliers system is

$$
\begin{aligned}
& 0=2\left(a+\frac{c_{n}}{2}\right) \lambda_{1}+2\left(a-\frac{c_{n}}{2}\right) \lambda_{2}+2 a \lambda_{3}, \\
& 0=2 b \lambda_{1}+2 b \lambda_{2}+2\left(b-\sqrt{1-\frac{c_{n}^{2}}{4}}\right) \lambda_{3},
\end{aligned}
$$




$$
\begin{aligned}
& 1=-2 p \lambda_{1}, \\
& 1=-2 q \lambda_{2} .
\end{aligned}
$$

Solving the two last equations for the Lagrange multipliers and substituting them into the two first ones gives

$$
\begin{aligned}
& q\left(a+\frac{c_{n}}{2}\right)+p\left(a-\frac{c_{n}}{2}\right)=2 a p q \lambda_{3} \\
& (q+p) b=2 p q\left(b-\sqrt{1-\frac{c_{n}^{2}}{4}}\right) \lambda_{3} .
\end{aligned}
$$

After eliminating $\lambda_{3}$ and making some simplifications, one gets

$$
(q-p) \frac{c_{n}}{2}\left(b-\sqrt{1-\frac{c_{n}^{2}}{4}}\right)=(q+p) a \sqrt{1-\frac{c_{n}^{2}}{4}} .
$$

Multiplying both sides by $(q-p)$ and using the constraints of (1) to substitute $(q+p)(q-p)=q^{2}-p^{2}=-2 a c_{n}$ leads to

$$
(q-p)^{2} \frac{c_{n}}{2}\left(b-\sqrt{1-\frac{c_{n}^{2}}{4}}\right)=-2 a^{2} c_{n} \sqrt{1-\frac{c_{n}^{2}}{4}} .
$$

Recall that we are looking for a solution with $b \geq \sqrt{1-\frac{c_{n}^{2}}{4}} \geq 0$ and $c_{n}>0$. Therefore, the left-hand-side term is nonnegative, and the right-hand-side term is nonpositive. To get an equality, we need that $a=0$ or that $a \neq 0$ and $c_{n}=2$.

- If $a=0$, then

$$
p=q=\sqrt{\frac{c_{n}^{2}}{4}+b^{2}}=\sqrt{\frac{c_{n}^{2}}{4}+\left(1+\sqrt{1-\frac{c_{n}^{2}}{4}}\right)^{2}}=\sqrt{2+2 \sqrt{1-\frac{c_{n}^{2}}{4}}}
$$

- If $a \neq 0$ and $c_{n}=2$, then (2) becomes $b(p-q)=0$. The case where $b=0$ corresponds to a minimizer, and $p=q$ implies that $(a+1)^{2}=(a-1)^{2}$, i.e., that $a=0$, which is a contradiction.

Therefore, we have shown that $c_{\ell-1}+c_{\ell} \leq 2 \sqrt{2+2 \sqrt{1-\frac{c_{n}^{2}}{4}}}$.

Combining the above lemmas leads to our main result.

Theorem 1 If $n$ is an odd integer, then the supremum of the perimeter in value over all simple polygons contained in a disk of radius one is

$$
p_{n}^{*}=\frac{\left(\sqrt{1+8(n-2)^{2}}-1\right)^{\frac{1}{2}}\left(\sqrt{1+8(n-2)^{2}}+3\right)^{\frac{3}{2}}}{4(n-2)},
$$

and the polygon is arbitrarily close to an isosceles triangle. 
Proof Combining Lemmas 3 and 4 and setting $v=\sqrt{1-\frac{c_{n}^{2}}{4}}$ leads to

$$
p_{n}^{*} \leq(n-2) c_{n}+2 \sqrt{2+2 \sqrt{1-\frac{c_{n}^{2}}{4}}}=2(n-2) \sqrt{1-v^{2}}+2 \sqrt{2+2 v}
$$

In order to minimize this upper bound, we take the derivative and set it equal to zero. This gives

$$
-2(n-2) \frac{v}{\sqrt{1-v} \sqrt{1+v}}+\frac{\sqrt{2}}{\sqrt{1+v}}=0
$$

and may be rewritten as $2(n-2)^{2} v^{2}+v-1=0$. The unique nonnegative solution is $v=\frac{-1+\sqrt{1+8(n-2)^{2}}}{4(n-2)^{2}}$. Substituting for $v$ and simplifying leads to

$$
p_{n}^{*} \leq \frac{\left(\sqrt{1+8(n-2)^{2}}-1\right)^{\frac{1}{2}}\left(\sqrt{1+8(n-2)^{2}}+3\right)^{\frac{3}{2}}}{4(n-2)} .
$$

A simple polygon with a perimeter arbitrarily close to that value can be constructed. Consider a simple polygon in which $(n-1)$ consecutive vertices alternate back and forth arbitrarily close to $\left(-\frac{c_{n}}{2}, 0\right)$ and $\left(\frac{c_{n}}{2}, 0\right)$, and the remaining vertex is located at $(0,1+v)$, where $c_{n}=2 \sqrt{1-v^{2}}$ and $v=\frac{-1+\sqrt{1+8(n-2)^{2}}}{4(n-2)^{2}}$. The resulting figure is arbitrarily close to an isosceles triangle, and in the limit, the perimeter satisfies

$$
p_{n}^{*}=\frac{\left(\sqrt{1+8(n-2)^{2}}-1\right)^{\frac{1}{2}}\left(\sqrt{1+8(n-2)^{2}}+3\right)^{\frac{3}{2}}}{4(n-2)} .
$$

In the case where $n=3$, the optimal triangle is equilateral. We conclude with the following corollary.

Corollary 1 For odd values of $n$, the sequence $\left\{\frac{p_{n}^{*}}{n}\right\}$ is monotone increasing.

Proof For any fixed value of $n$, maximizing the perimeter or maximizing the average edge length yields the same figure. Furthermore, for any fixed odd value of $n$, one can easily construct an $(n+2)$-sided polygon whose average edge length strictly exceeds $\frac{p_{n}^{*}}{n}$. Indeed, one simply has to consider an optimal $n$-sided polygon and add a vertex near $(-a, 0)$ and another one near $(a, 0)$. This will add two edges of length arbitrarily close to the value $c_{n}>\frac{p_{n}^{*}}{n}$.

\section{References}

1. Brass, P., Moser, W., Pach, J.: Research Problems in Discrete Geometry. Springer, New York (2005) 